\title{
The Rate of Convergence of Chebyshev Polynomials for Functions Which Have Asymptotic Power Series About One Endpoint
}

\author{
By John P. Boyd*
}

\begin{abstract}
The theorem proved here extends Chebyshev theory into what has previously been no man's land: functions which have an infinite number of bounded derivatives on the expansion interval $[a, b]$ but which are singular at one endpoint. The Chebyshev series in $1 / x$ for all the familiar special functions fall into this category, so this class of functions is very important indeed.

In words, the theorem shows that the more slowly the asymptotic power series about the singular point converges, the slower the convergence of the corresponding Chebyshev series must be. More formally, if $f(x)$, analytic on $[a, b)$, is singular at $x=b$ in such a way that it has an asymptotic power series $f(x) \sim \sum a_{n}(x-b)^{n}$ about that endpoint, then, if

$$
\varlimsup_{n \rightarrow \infty} \frac{\log \left|a_{n}\right|}{n \log n}=r \text {, }
$$

it is proved that the coefficients of the convergent Chebyshev polynomial series on $[a, b]$, $f(x) \sim \sum b_{n} T_{n}(y)$ where $y=2[x-0.5(b+a)] /(b-a)$, satisfy the inequality

$$
\varlimsup_{n \rightarrow \infty} \frac{\log \left|\left(\log \left|b_{n}\right|\right)\right|}{\log n}<\frac{2}{r+2} .
$$
\end{abstract}

It is well known that if a function $f(x)$ is singular about a point, let us say $x=1$ to be definite, then the power series of $f(x)$ about that point is at most asymptotic and must diverge. The corresponding series of Chebyshev polynomials on $[-1,1]$, which includes the singularity as an endpoint, is much more robust. If the singularity is weak, in the sense that all (left) derivatives of $f(x)$ are bounded at $x=1$, then it can be proven by a simple integration-by-parts argument [1] that the Chebyshev series converges absolutely and exponentially on the interval. Indeed, such series, usually in the form of series of shifted Chebyshev polynomials in $1 / x$, are enormously useful in approximation theory for representing the large $x$ behavior of Bessel functions and many other common transcendentals. Luke [2] gives extensive tables of such approximations.

Although a Chebyshev series, whose coefficients are $O\left(e^{-\lambda n^{8}}\right)$, technically possesses the property of exponential or "infinite order" convergence for any positive $\beta$, the precise value of $\beta$ is obviously of great practical importance. The integrationby-parts argument implies only that $\beta>0$. When a function has a singularity which is not on $[-1,1]$, it is known that $b_{n} \sim \alpha e^{-\lambda n}$ for some $\alpha$ and $\lambda$ which depend

Received March 21, 1980; revised August 21, 1980 and October 29, 1980.

AMS (MOS) subject classifications (1970). Primary 42A56; Secondary 33A65, 41A10.

Key words and phrases. Chebyshev polynomial series.

- Current address: Department of Atmospheric and Oceanic Sciences, University of Michigan, 2455 Hayward Avenue, Ann Arbor, Michigan 48109. 
on the location and residue of the singularity [1] (i.e., the coefficients form a geometric series, at least asymptotically); when the singularity is at an endpoint, this argument implies that the convergence must be "subgeometric," i.e., $\beta<1$. The goal of this work is to prove a tighter bound on $\beta$.

For simplicity, it shall be assumed throughout that the singularity is at $x=1$ and that the desired interval of Chebyshev expansion is $[-1,1]$. However, these ideas can be generalized to an arbitrary interval $[a, b]$, with the singularity at $x=b$, merely by making the simple linear change of variable from $x \in[a, b]$ to $y \in$ $[-1,1]$ which is given in the abstract.

The first step in the proof is to formally define the two orders of convergence which the theorem will compare.

Definition. The order $r$ of an asymptotic series $\sum a_{n}(x-b)^{n}$ about the point $x=b$ is defined by

$$
r \equiv \varlimsup_{n \rightarrow \infty} \frac{\log \left|a_{n}\right|}{n \log n} .
$$

Thus, the series $\Sigma(-1)^{n} n ! x^{n}$ has order 1 while $\Sigma(2 n) ! x^{n}$ and $\Sigma(n !)^{2} x^{n}$ both have $r=2$. The greater the order of the series of $f(x)$ about a given point, the more rapidly its derivatives at the chosen point increase with increasing degrees of differentiation, i.e., if $b=1$, then

$$
\varlimsup_{n \rightarrow \infty} \frac{\log \left|f^{(n)}(1)\right|}{n \log n}=r+1,
$$

from Taylor's theorem.

Strictly speaking, one must distinguish between left- and right-hand derivatives in (2), but this is usually obvious from the context. In the rest of the paper, $f(x)$ is assumed to be analytic on $[-1,1]$, except at $x=1$, so clearly (2) should be interpreted as the derivative as $x \rightarrow 1^{-}$.

Definition. The index of exponential convergence $\beta$ of the Chebyshev series $\sum b_{n} T_{n}(x)$ is the least upper bound of those $k$ for which

$$
\left|b_{n}\right| \leqslant p e^{-q n^{k}}
$$

can be satisfied for all $n$ with some finite positive constants $p$ and $q$.

This definition implies that, for any function $f(x)$ of index of convergence $\beta$, $\beta>0$ (as henceforth assumed), one can find a function $R(x ; p, q, k)(k<\beta)$ defined by

$$
R(x ; p, q, k)=p \sum_{n=0}^{\infty} e^{-q n^{k}} T_{n}(x)
$$

which, term-by-Chebyshev-term, is greater than $f(x)$ since the Chebyshev polynomials satisfy the bound $\left|T_{n}(x)\right| \leqslant 1$ for all $n$ and all $x \in[-1,1]$. This in turn implies

$$
R^{(n)}(1) \geq\left|f^{(n)}(1)\right|
$$

for all $n$. The grand strategy of our proof will be to estimate $R^{(n)}(1 ; p, q, k)$ as a function of $n$ and $k$ and $f^{(n)}(1)$ as a function of $n$ and $r$. We will then show that, unless $k \leqslant 2 /(r+2)$, the derivatives of $f(x)$ at $x=1$ will grow faster with $n$ than those of the bounding function $R(x ; p, q, k)$, which, according to (5), is impossible. 
Since (2) already provides all the needed information on $f^{(n)}(1)$, the principal task of the proof of the theorem is to estimate the magnitude of $R^{(n)}(1)$, which will be done by Lemma 3. In order to make this estimate, however, we need to establish two simpler results first.

\section{LEMMA 1.}

$$
T_{j}^{(n)}(1)=\frac{\pi^{1 / 2} j \Gamma(j+n)}{\Gamma(j-n+1) 2^{n} \Gamma(n+1 / 2)} .
$$

Proof. The identity

$$
T_{j}^{(n)}(1)=\prod_{k=0}^{n-1} \frac{\left(j^{2}-k^{2}\right)}{(2 k+1)}
$$

is given by Gottlieb and Orszag [1]. By factoring $\left(j^{2}-k^{2}\right)$ and repeatedly using the definition of the gamma function, (7) can be rearranged into (6).

LEMMA 2. Let the function $S(n, q, k)$ be defined by the sum

$$
S \equiv \sum_{j=0}^{\infty} e^{-q j^{k} j^{2 n}}
$$

Then

$$
\log S(n, q, k) \leqslant \frac{2 n}{k} \log (n)+O(n) .
$$

Proof. The sum can be interpreted as the exact integral of the piecewise continuous function defined by

$$
g(x)=e^{-q j^{k} j n}, \quad j<x<j+1,
$$

$$
S=\int_{0}^{\infty} g(x) d x
$$

Since the integrand of

$$
I(n, q, k) \equiv \int_{0}^{\infty} e^{-q(x+1)^{k}}(x+1)^{2 n} d x
$$

is everywhere greater than $g(x)$, it follows that

$$
S \leqslant I \text {. }
$$

By making the change of variable $x+1 \rightarrow u$,

$$
\begin{aligned}
I & =\int_{1}^{\infty} e^{-q u^{k} u^{2 n} d u} \\
& =\int_{0}^{\infty} e^{-q u^{k}} u^{2 n} d u-\int_{0}^{1} e^{-q u^{k} u^{2 n}} d u
\end{aligned}
$$

The first integral in (15) is (with a change of variable) the integral definition of the gamma function while the second can be bounded by

$$
\int_{0}^{1} u^{2 n} d u
$$

which is obviously $1 /(2 n+1)$. Thus,

$$
I=\frac{1}{k} \Gamma\left(\frac{2 n+1}{k}\right) q^{-(2 n+1) / k}+O\left(n^{-1}\right) .
$$

Applying Stirling's formula to (16) then gives (9). 
Lemma 3. Let $p, q$ be positive constants and let $0<k<1$. Then

$$
\varlimsup_{n \rightarrow \infty} \frac{\log R^{(n)}(1 ; p, q, k)}{n \log n}<\left(\frac{2}{k}-1\right) .
$$

Proof. From (4) and Lemma 1, one has (using $R$ for $R^{(n)}(1 ; p, q, k)$ )

$$
R=Q \sum_{j=0}^{\infty} e^{-q^{k}} \frac{j \Gamma(j+n)}{\Gamma(j-n+1)},
$$

where

$$
Q=\frac{\pi^{1 / 2} p}{2^{n} \Gamma(n+1 / 2)}
$$

Now

$$
\begin{aligned}
\frac{j \Gamma(j+n)}{\Gamma(j-n+1)} & =\frac{j \Gamma(j+2 n)}{\Gamma(j-n+1)[(j+n)(j+n+1) \cdots(j+2 n-1)]} \\
& <\frac{j \Gamma(j+2 n)}{j !},
\end{aligned}
$$

where the last expression follows by replacing each of the factors of $j+n+i-1$ in the square brackets in (20) by the smaller factor $j-n+i(i=1,2, \ldots, n)$. Cancelling the common factors shows that

$$
\begin{aligned}
\frac{j \Gamma(j+2 n)}{j !} & =j(j+1) \cdots(j+2 n-1) \\
& <(j+2 n)^{2 n},
\end{aligned}
$$

where (23) follows by replacing each of the $2 n$ factors in (22) by $(j+2 n)$. Combining (20) through (23) with (18) shows

$$
R<Q \sum_{j=0}^{\infty} e^{-q j^{k}}(j+2 n)^{2 n}
$$

Since

$$
j^{k}+(2 n)^{k}>(j+2 n)^{k}, \quad 0<k<1,
$$

it follows that

$$
R<Q e^{q(2 n)^{k}} \sum_{j=0}^{\infty} e^{-q(j+2 n)^{k}}(j+2 n)^{2 n}
$$

Since the sum in (25) is equivalent to that defining $S$ (Eq. (9)), but with the first $2 n$ terms omitted, it is clear that, using the definition of $Q$,

$$
R<\frac{\pi^{1 / 2} p e^{q(2 n)^{k}}}{2^{n} \Gamma(n+1 / 2)} S(n, q, k) .
$$

Lemma 2 and Stirling's formula show

$$
R<\left(\frac{2}{k}-1\right) n \log n+O(n),
$$

and the lemma follows immediately. 
Armed with estimates of $R^{(n)}(1 ; p, q, k)$, it is easy to prove

THEOREM. If $f(x)$ is a function which is analytic on $[-1,1]$, except for a singularity at $x=1$ where it has an asymptotic power series $f(x) \sim \sum a_{n}(x-1)^{n}$ whose order of convergence is $r$ where $r$ is defined by

$$
r \equiv \varlimsup_{n \rightarrow \infty} \frac{\log \left|a_{n}\right|}{n \log n}
$$

then the index of exponential convergence $\beta$ of its Chebyshev series, where $\beta$ is defined as the least upper bound of $k$ such that

$$
\left|b_{n}\right| \leqslant p e^{-q n^{k}}
$$

for some positive constants $p$ and $q$ and all $n$, must satisfy

$$
\beta \leqslant \frac{2}{r+2},
$$

assuming this index of convergence exists.

The theorem can be extended to intervals other than $[-1,1]$ by a trivial change of variables.

Proof. For every $0<k<\beta$,

$$
\left|f^{(n)}(1)\right| \leqslant R^{(n)}(1 ; p, q, k)
$$

for some positive $p, q$ where the left-hand derivative is implied. Thus,

$$
\begin{aligned}
1+r & =\varlimsup_{n \rightarrow \infty} \frac{\left|f^{(n)}(1)\right|}{n \log n} \\
& \leqslant \frac{2}{k}-1,
\end{aligned}
$$

where the inequality follows from (31) and Lemma 3. Taking the least upper bound of $k$ gives

$$
1+r<\frac{2}{\beta}-1
$$

which is equivalent to (30).

A few comments on the theorem are in order. First, the restriction that $f(x)$ be analytic on $[-1,1]$, except at an endpoint, has been imposed because it is well known [1] that, if the $j$ th derivative of $f(x)$ is unbounded at some point on the interior of $[-1,1]$, then

$$
b_{n} \sim O\left(n^{-(j+1)}\right)
$$

and $\beta>0$ is impossible. In this event, the only sensible course is to speak of $j$ in (35) as the index of algebraic convergence for the Chebyshev series and to dump the theorem proved here in favor of these earlier results [1] relating $j$ to the degree of the lowest derivative which is unbounded.

Second, the restriction that $f(x)$ be singular at an endpoint is also necessary for the theorem to improve upon past work. If $f(x)$ is analytic on all of $[-1,1]$ but has a singularity somewhere else in the complex plane, then the index of exponential 
convergence $\beta=1$, and one can in fact obtain the precise asymptotic form of the Chebyshev coefficients if the type and location of the singularity nearest to $[-1,1]$ is known [1].

It should not be inferred, however, that functions which meet these restrictions are rare or exotic. All special functions which have asymptotic power series in $1 / x$ have corresponding Chebyshev expansions in $1 / x$ which meet the conditions of the theorem. Thus, the theorem applies to almost half the series in Luke's treatise [2], which is the most extensive collection of numerical Chebyshev series to date.

Third, this theorem is similar both in spirit and proof to the well-known theorem of complex variable theory which relates the $\overline{\lim }\left|a_{n}\right| /(n \log n)$ of the power series coefficients of an entire function to its order. Here, we compare growth of derivatives with $n$ to the Chebyshev coefficients rather than growth of the function itself with $|x|$ to the power series coefficients, but the basic idea is the same: the faster the growth in the property of $f(x)$, the slower the series must converge in order to reproduce this growth. The Hermite polynomial convergence theorem of Boyd [4] is also similar.

Fourth, one can of ten show that the inequality in the theorem can be replaced by an equals sign in specific cases. The Stieltjes function, for example, which is defined by

$$
f(x) \equiv \int_{0}^{\infty} \frac{e^{-t} d t}{1+x t}
$$

has the asymptotic series

$$
f(x) \sim \sum(-1)^{n} n ! x^{n}
$$

and is therefore of convergence order $r=1$ at $x=0$. This implies that $\beta<2 / 3$, according to the theorem, but Luke [2] has shown that the Chebyshev expansion of (36) on $[0,1]$ has

$$
b_{n} \sim O\left(e^{-3 n^{2 / 3}}\right)
$$

i.e., $\beta=2 / 3$ precisely.

This raises the obvious question: can we strengthen the theorem for all cases by replacing the equality by an equals sign? The answer is no; a simple counterexample is provided by $f(x)=e^{-1 / x^{2}}$. Its (right-hand!) derivatives at $x=0$ are all zero, implying that $r=0$, but, by applying the method of steepest descents to the coefficient integrals, it can be shown [5] that $\beta=2 / 3$ rather than the upper bound of $\beta=1$ given by the theorem.

Nonetheless, it seems reasonable that there does exist a general class of functions for which $\beta=2 /(r+2)$-perhaps it includes all functions which have nontrivial, i.e., nonzero, asymptotic series about the singular point. The obvious goal of future research is to strengthen the results of this paper by (i) determining when the inequality can be replaced by an equals sign and (ii) obtaining information about the constants $p$ and $q$, i.e., more precise information about the convergence of the series than that given by $\beta$ alone. For individual functions, steepest descent can be very useful in finding the asymptotic form of the $b_{n}[5]$. 
Acknowledgement. This work was supported by NASA Grant NGL-22-007-228. I thank the referee for his helpful comments, especially for his suggested proof of Lemma 3.

The Center for Earth and Planetary Physics

Harvard University

Cambridge, Massachusetts 02138

1. D. GotTlieb \& S. A. OrszAg, Numerical Analysis of Spectral Methods: Theory and Applications, SIAM, Philadelphia, Pa., 1977.

2. Y. Luke, The Special Functions and Their Approximations, Vol. II, Academic Press, New York, 1969.

3. M. Abramowttz \& I. Stegun, Eds., Handbook of Mathematical Functions, Dover, New York, 1965.

4. J. BoYD, "The rate of convergence of Hermite function series," Math. Comp., v. 35, 1980, pp. 1309-1316.

5. J. BoyD, "The optimization of convergence for Chebyshev polynomial methods in an unbounded domain," J. Comput. Phys. (To appear.) 